\title{
HOW CAN ATTOSECOND PULSE TRAIN INTERFEROMETRY interrogate electron dynamics?
}

\author{
C.L. ARNOLD, M. ISINGER, \\ D. BUSTO, D. GUÉNOT, \\ S. NANDI, S. ZHONG, \\ J.M. DAHLSTRÖM, \\ M. GISSELBRECHT, \\ A. L'HUILLIER \\ Department of Physics, \\ Lund University, Lund, Sweden \\ cord.arnold@fysik.lth.se
}

Light pulses of sub-100 as ( 1 as $\left.=10^{-18} \mathrm{~s}\right)$ duration, with photon energies in the extreme-ultraviolet (XUV) spectral domain, represent the shortest event in time ever made and controlled by human beings. Their first experimental observation in 2001 has opened the door to investigating the fundamental dynamics of the quantum world on the natural time scale for electrons in atoms, molecules and solids and marks the beginning of the scientific field now called attosecond science.

is reached. Depending on the generation conditions, the cut-off can exceed $100 \mathrm{eV}$ of photon energy, covering several tens of harmonics, and the effect was thus named high-order harmonic generation (HHG). It was soon realized that the comb of harmonics could correspond to a train of very short pulses, i.e. attosecond pulses, if the harmonics were phase-locked [3]. This view was inspired by a semi-classical model of the single-atom response in a strong driving laser field [4], which was shortly after also supported by a fully quantum mechanical treatment $[5,6]$. until a sharp drop, called the cut-off,

ttosecond light pulses cannot be obtained from a convenfemtosecond laser pulses are focused into a dilute gas. It was observed in the late eighties that this interaction leads to the emission of a comb of odd-order harmonics of the driving laser $[1,2]$. Unexpected from the concepts of perturbative nonlinear optics, only the first few orders decrease exponentially in power, while higher orders form a plateau of almost equal power

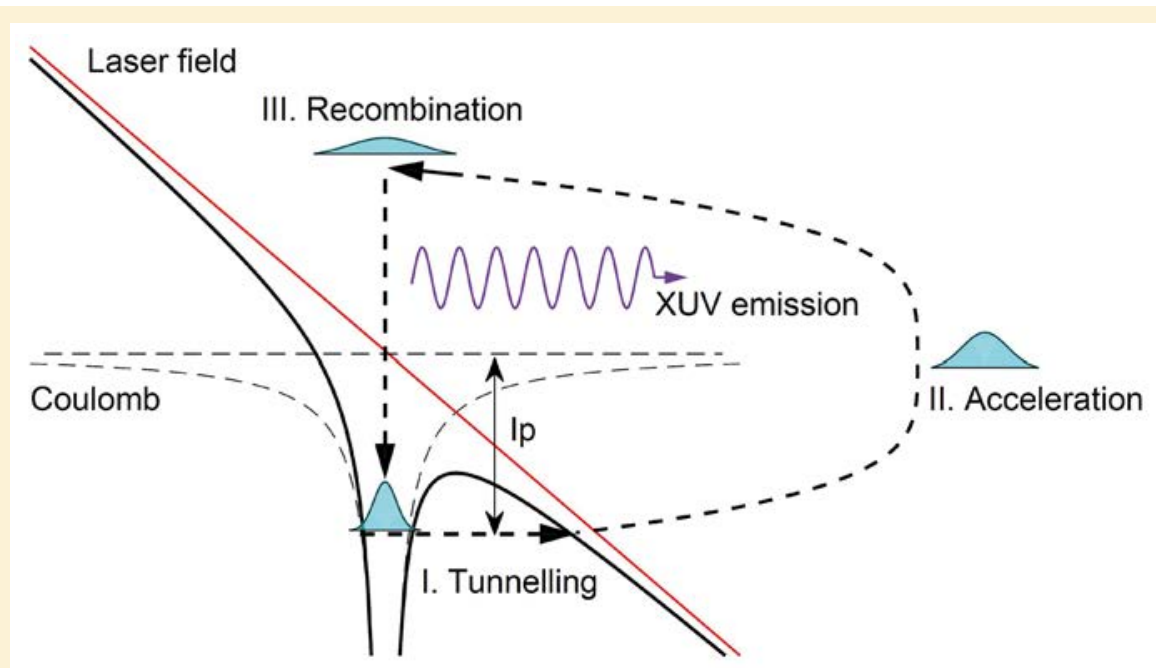

Figure 1. Illustration of the semi-classical three-step model for high-order harmonic generation.
The semi-classical understanding of HHG, generally referred to as the three-step model, is illustrated in Figure 1. First, the atomic binding potential is so strongly distorted near the crests of the driving laser field that the least bound electron may tunnel-ionize to the continuum. Second, driven by the strong laser field, the electron is taken away from the parent-ion, picking up kinetic energy. Finally, when the driving field changes sign, the electron may return to its parent-ion and recombine, whereas its excess energy is emitted as an XUV photon. The kinetic energy of the returning electron depends on its trajectory, i.e. the path it takes from the time it was born in the continuum to its return to the parent-ion. Not all possible trajectories return to the parent-ion and contribute to HHG. The three-step process repeats itself for every half cycle of the driving field, resulting in an attosecond pulse train (APT). While the spectrum of each individual attosecond pulse in the train is continuous, the corresponding spectrum of the train results from the spectral interference of all pulses in the train and is composed of odd-order harmonics. This can be understood in analogy to the frequency comb structure 
of the output of an ultrafast oscillator, where the interference of the output pulses results in comb lines spaced by $1 / f$, where $f$ is the repetition rate of the oscillator. Single attosecond pulses (SAPs) can be obtained by spatially separating the pulses in the train $[7,8]$ or by manipulating the driving pulse in a way that the interaction is driven by only one half-cycle of the field [9]. The conversion efficiency for HHG, i.e. the ratio of the energy of the attosecond pulse train or single attosecond pulse to the energy of the driving laser pulse, is about $10^{-5}$ at best (usually lower for single attosecond pulses and for photon energies larger than $50 \mathrm{eV}$ ), determined both by the single-atom response and by phase-matching in the generation gas. Still, modern attosecond pulse sources can have average powers in the range of $\mu \mathrm{W}$ to $\mathrm{mW}$ [10].

After the first observation of HHG, it took almost fifteen more years until the duration of attosecond pulses in a train as well as that of a single attosecond pulse were finally experimentally measured in 2001 [11,12]. The measurement approaches, i.e. RABBIT (Reconstruction of Attosecond Bursts by Interference of Two-photon Transitions) for APTs and the Attosecond Streak Camera for SAPs, are based on performing cross-correlations of the APT or SAP with a longer low-frequency pulse, usually a copy of the driving pulse for HHG, while the photoelectron spectrum originating from a detection gas as a result of the two fields is recorded. The spectral amplitude and phase of the APT or SAP are encoded in the photoelectron spectrum and the pulses can be retrieved with different computer algorithms.

\section{The RABBIT technique for characterizing attosecond pulse trains}

In this article, we will focus on the RABBIT technique and how it can be used to learn about fundamental electron dynamics on the attosecond time scale. For that, we will first discuss the RABBIT scheme in more detail. The principle is illustrated in Figure 2. APTs synchronized with a weak copy of the driving pulse, in the following referred to as probe pulse, are sent into a photoelectron spectrometer, where photoelectrons are generated from a detection gas (usually noble gases), while the time delay between the APTs and the probe field is scanned with interferometric precision. Employing photoelectron spectroscopy for measuring attosecond pulses is somewhat obvious, taking into account that the photon energy generally overcomes the ionization potential of neutral gases. The underlying idea of RABBIT is to measure the phase difference between consecutive harmonics, which is the information needed to reconstruct the average attosecond pulse in the train. In optics, a phase difference is often assessed by interference. The different harmonics of the frequency comb representing an APT do however not result in a steady and observable interference, because they are separated in photon energy, so that the generated photoelectrons are also well separated in kinetic energy. The probe pulse however couples consecutive harmonics by introducing sidebands to the photoelectron spectrum, which are located between the harmonics. The sidebands are due to two-colour two-photon ionization; either a harmonic and a probe photon are absorbed simultaneously or a photon from the next harmonic is absorbed and a probe photon is emitted, resulting in two possible quantum paths from two consecutive harmonics to the same sideband, thus leading to interference. The sidebands oscillate with the time delay $\tau$ between the APT and the probe field as

$$
S_{2 q}=\alpha+\beta \cos \left[2 \omega \tau-\Delta \phi_{2 q}-\Delta \theta_{2 q}\right]
$$

where $\alpha$ and $\beta$ describe the amplitude and contrast of the oscillations, respectively and $\Delta \phi_{2 \mathrm{q}}=\phi_{2 \mathrm{q}+1}-\phi_{2 \mathrm{q}-1}$ is the phase difference between the consecutive harmonics of the orders $2 q+1$ and $2 q-1$, where $q$ is an integer. 
(a) |g> HH15 SB16 HH17 SB18 HH19

(b)

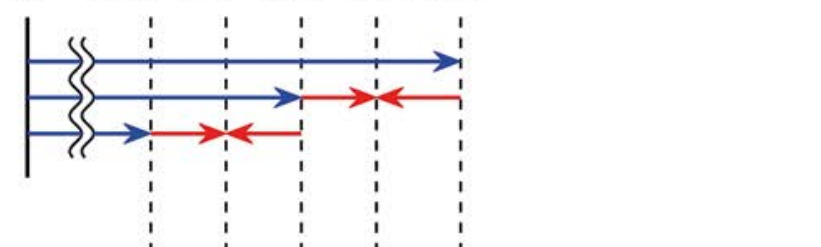

(c)
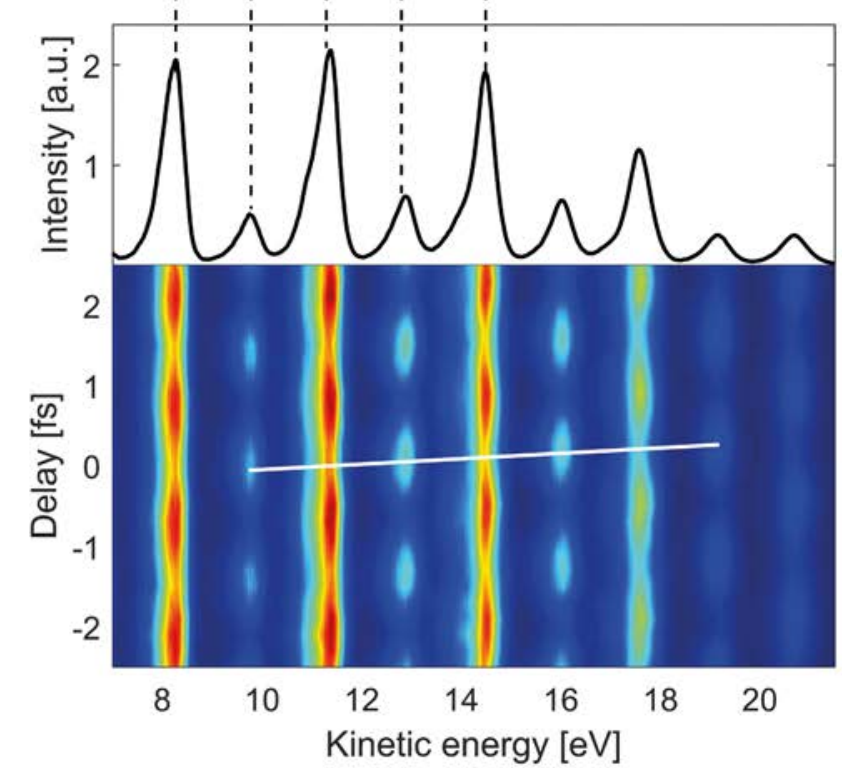

(d)

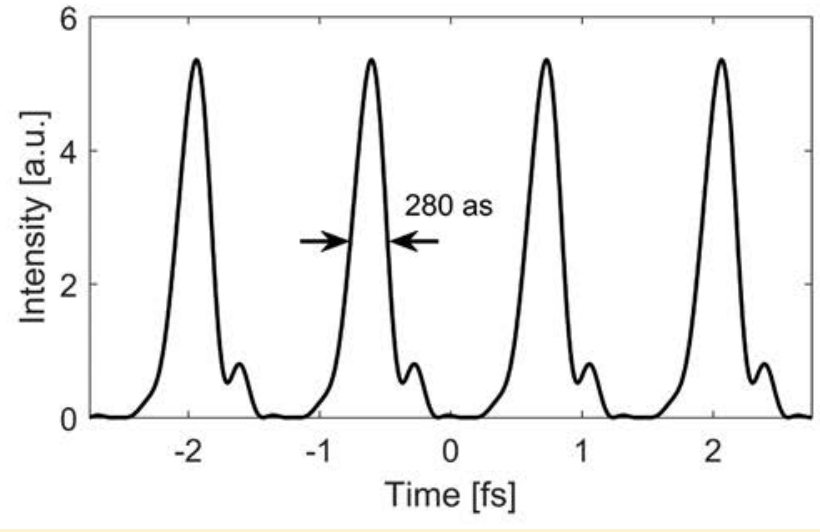

Figure 2.

Illustration of the RABBIT

technique. Panel (a) shows how two-colour (APT + probe) ionization leads to the formation of sidebands between the harmonics (blue) in the photoelectron spectrum plotted in (b). Panel (c) shows a RABBIT

trace, i.e.

photoelectron spectrum $v$ s. delay between the APTs and probe pulses. The white line indicates the measured phase differences between

harmonics, encoded in the sideband phases, which allow for reconstruction of the average pulse in the APT, as shown in (d).

The last term, $\Delta \phi_{2 q}$, often referred to as atomic phase, is an intrinsic contribution from the detection gas due to two-colour ionization. If the phase differences between all consecutive harmonics are known, the average attosecond pulse in the train can be obtained by coherently adding the harmonics with their respective phase offsets. It should be noted that this is only accurate if the atomic contribution, i.e. $\Delta \phi_{2 q}$, is small compared to the phase differences between the harmonics. Among many achievements, the RABBIT technique has shown that the intrinsic chirp of attosecond pulses, which often is positive, can be compensated by transmission through thin metallic foils that provide anomalous dispersion in the XUV spectral range [13].

\section{Investigating electronic dynamics on the attosecond time scale}

While the early days of attosecond science were mostly dedicated to the characterization of the spectral and temporal properties of attosecond pulses, the focus has later shifted towards actually applying those pulses for studying dynamics on a time scale that was not accessible before. One of the most prominent questions in this respect is "how long does ionization take?", i.e. "how long does it take for a photoelectron to actually leave the atom after interacting with an attosecond pulse?" The contrary question, i.e. "how long would it take the parent-atom or molecule to know that it has been ionized and become an ion?" is equally intriguing. However, since the most prominent experimental tools of the field, i.e. streaking and RABBIT, inherently employ photoelectron spectroscopy, the leaving of a photoelectron is more straightforward to study. It should however be noted that the ionization time is a delicate quantity to define. After ionization, the photoelectron moves in the Coulombic potential of the ion, which changes with the inverse of the distance to the ion and formally reaches infinitely far; any definition of when the photoelectron has left the proximity of the ion would be somewhat arbitrary. What helps here, is a more fundamental quantum mechanical view on ionization, where we consider photoelectron wave packets of Coulombic waves instead of a classical particle-like understanding of photoelectrons. An electron wave packet is a quantum mechanical construction, describing the electron's probability amplitude to be found in a certain position at a certain time. After ionization the photoelectron wave packet moves in the potential landscape of the ion. Similarly to an ultrashort laser pulse propagating in a dispersive medium, where the speed is a function of wavelength, the electron wave packet will pick up a group delay, which is defined as the derivative of its phase in respect to energy. While the Coulomb potential formally reaches infinitely far, the group delay of an electron wave packet propagating through it is finite. The acquired group delay can be interpreted as ionization time. This view was first introduced by Wigner for scattering events [14]. 


\section{Delivering advanced solutions for fiber lasers from 0.8 to $2.1 \mu \mathrm{m}$.}

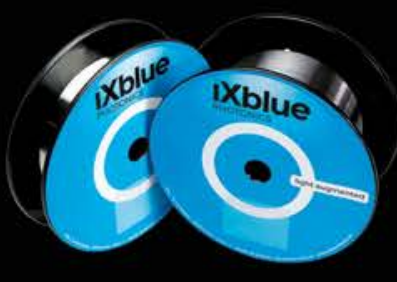

\section{Doped Fibers}

- LMA \& PM versions

- Advanced coating

- Matched passive fibers

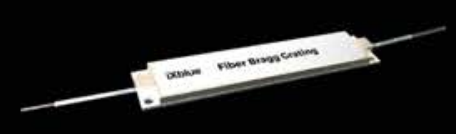

FBG mirrors

- Custom profile

- High power handling

- Dissipative package
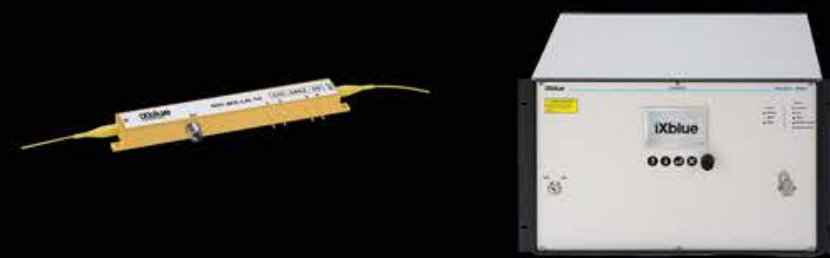

\section{$\mathrm{LiNbO}_{3}$ modulators Modbox}

- Very low insertion loss

- Wide modulation

bandwidth

- High extinction ratio
- Sub-ns to longer optical pulses

- Pulse picker / Pulse Shaping / Front-End

- Turn-key and

Optimized Solutions

\section{iXblue}




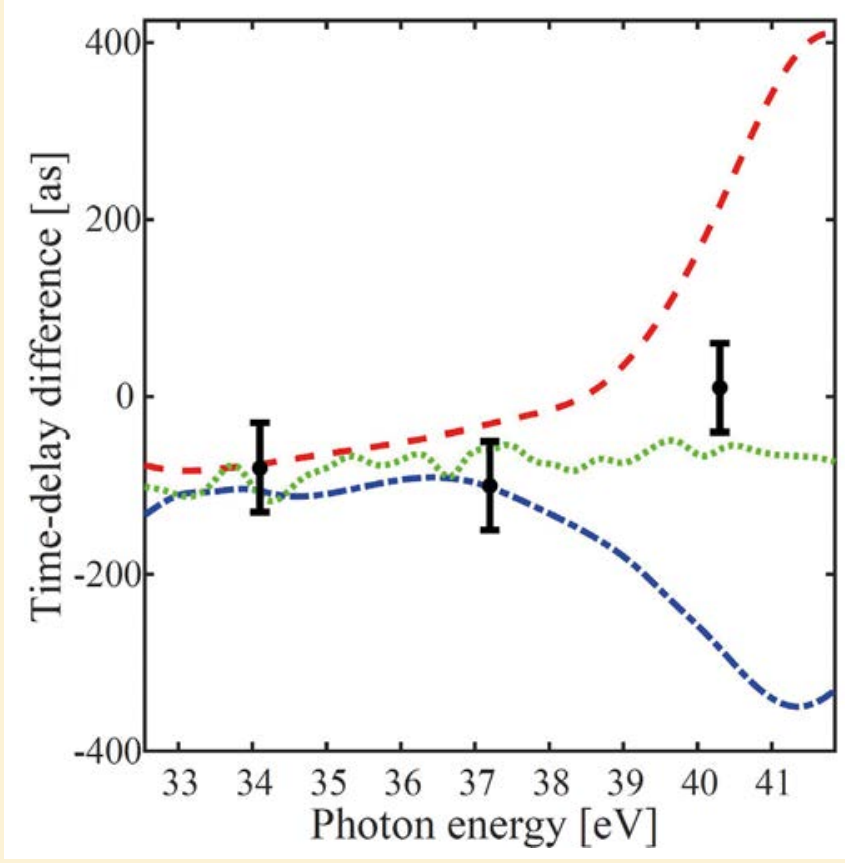

Figure 3. Measured relative ionization time delay between the $3 s$ and $3 p$ subshells in argon in comparison with different calculation methods. The figure is adapted from [18] with additional calculations from [19].

Determining the absolute ionization time however is complicated. Experiments have therefore mostly focused on measuring the relative time delay between photoelectron wave packets from different initial states, which is an easier question to answer than the absolute time delay. Prominent early examples of such experiments are the measurement of a relative time delay of about 100 as between the ionization from valence- and conduction band states in tungsten [15] as well as the relative time delay of $21 \pm 5$ as that was measured for ionization from the $2 p$ subshell in neon as compared to the $2 s$ sub-shell [16]. The latter work, further stimulated by the intriguing fact that the magnitude of the observed time delay could not be reproduced theoretically, has triggered a vast number of experimental and theoretical efforts to understand the origin of ionization time delays, and assessing such time delays became an important direction in attosecond science throughout the last few years.

While the pioneering experiments mentioned above were performed with the streaking technique, the first attosecond ionization time delays investigated with RABBIT were measured in the $n=3$ shell in argon
$[17,18]$. The energy range around $40 \mathrm{eV}$ that was investigated is particularly interesting because due to strong electron correlation effects, i.e. interactions between the different electrons of the atom, this energy region is difficult to treat theoretically. Thus, the measured difference in time delay can serve as qualitative indicator for the suitability of theoretical models. To illustrate how the RABBIT technique can be used to assess time delays, we shall rewrite equation 1 as

$$
S_{2 q}=\alpha+\beta \cos \left[2 \omega\left(\tau-\tau_{2 q}-\tau_{\theta}\right)\right],
$$

where we express the phase differences as finite difference approximations of group delays, i.e. $\tau_{2 q}=\left.\frac{\partial \phi}{\partial \Omega}\right|_{\Omega=2 q \omega} \approx \frac{\Delta \phi_{2 q}}{2 \omega}$ and $\tau_{\theta} \approx \frac{\Delta \theta_{2 q}}{2 \omega}$, where $\omega$ is the carrier frequency of the laser pulses. In this view, the sideband oscillations described by equations 1 and 2, respectively can be interpreted differently. As ionization happens, the electron wave packet inherits the group delay of the harmonics, which explains one contribution to the phase of the RABBIT sidebands, i.e. the one corresponding to $\tau_{2 q}$. The other contribution, $\tau_{\theta}$, refers to a delay that the electron wave packet acquires in the potential landscape of the ion in the presence of the probe field, where the contribution from the probe can often be determined theoretically [20]. While the contribution from $\tau_{\theta}$ in the past was considered small, it has now moved into the focus for measuring photoionization time delays. However, determining absolute delays remains difficult since the $a b$ solute phase of a RABBIT sideband depends on the delay between the APT and the probe pulse, which usually is not known accurately enough. Thus, relative time delay measurements are performed. For example in the case of the photoionization in the $n=3$ shell in argon, two RABBIT traces are recorded simultaneously for photoelectrons originating from the $3 p$ and from the $3 s$ sub-shells. As the photoelectrons are generated from identical attosecond pulses, any relative shift of the sidebands in the two RABBIT traces must originate from different group delays that the respective electron wave packets experience as a result of two-colour ionization. Figure 3 shows measured relative time delays in comparison to different theoretical models. The different theories differ significantly around $40 \mathrm{eV}$ and none of them shows perfect agreement with the measured delays.

It is fascinating to note that the relative time delays extracted from RABBIT traces are usually much smaller than the pulse duration of the attosecond pulses used in the measurement. The minimum observable delay is limited to how accurately the phase of the sidebands can be determined. This depends on the signal-to-noise ratio and the stability of the interferometer controlling the delay between the APTs and probe pulses rather than on the duration of the attosecond pulses. It is a very common feature of interferometric measurements, where often the phase of interferometric fringes can be determined with much greater accuracy than the wavelength of the light. One prominent example for this are gravitational wave detectors. 
A proof-of-principle experiment performed in xenon showed that the RABBIT technique could be applied for more complicated ionization processes than those discussed so far [21]. In one-photon double-ionization, a single absorbed XUV photon leads to the ejection of two photoelectrons which share the excess energy continuously. The two electrons must thus interact with each other resulting in a time delay. Incorporating the ion into the picture, this is the prototype of the quantum mechanical three-body problem and therefore extremely interesting to study. However, recording a RABBIT trace in this case is much more challenging because the pairs of correlated photoelectrons must be measured in coincidence. This means, to be sure that two detected electrons originate from the same ionization event, one has to work at a rate of less than one event per laser shot, which makes recording RABBIT traces very time consuming and puts large demands on the laser's long-term stability. Comparing to single ionization from the $5 p$-shell, which is recorded simultaneously, the relative ionization time delay for the double-ionization process could be extracted [21]. Here, single ionization was used as a reference clock, because the absolute phase of RABBIT sidebands, as discussed earlier, is usually unknown.

\section{Further developments}

Since the first RABBIT measurements, femtosecond laser technology has evolved rapidly. One interesting aspect of that development is spectral tunability. Employing tunability of the carrier wavelength of the driving pulses for HHG, the high-order harmonics are no longer fixed at specific photon energies. If one harmonic is tuned through a resonance, the phase of that resonance will be carried into the sidebands above and below that harmonic. The respective next sidebands will however be unaffected and can serve as reference to extract the phase associated with the resonance. This technique was applied to study the phase in two-colour two-photon ionization of helium [22] and more recently to measure the phase evolution of a Fano resonance in argon [23]. Fano resonances are a very general phenomenon in physics, characterized by an asymmetric line shape that originates from the interference between a resonant process and a background [24]; in atomic systems, between direct photoionization to the continuum and excitation to a quasi-bound state above the ionization potential, which will rapidly decay (within femtoseconds) to the
Figure 4. Phase variation of sideband 16 plotted against the photon energy of harmonic 17, which is scanned through the Fano resonance. The black circles are the measurement and the red line shows calculations. The figure is adapted from [23].

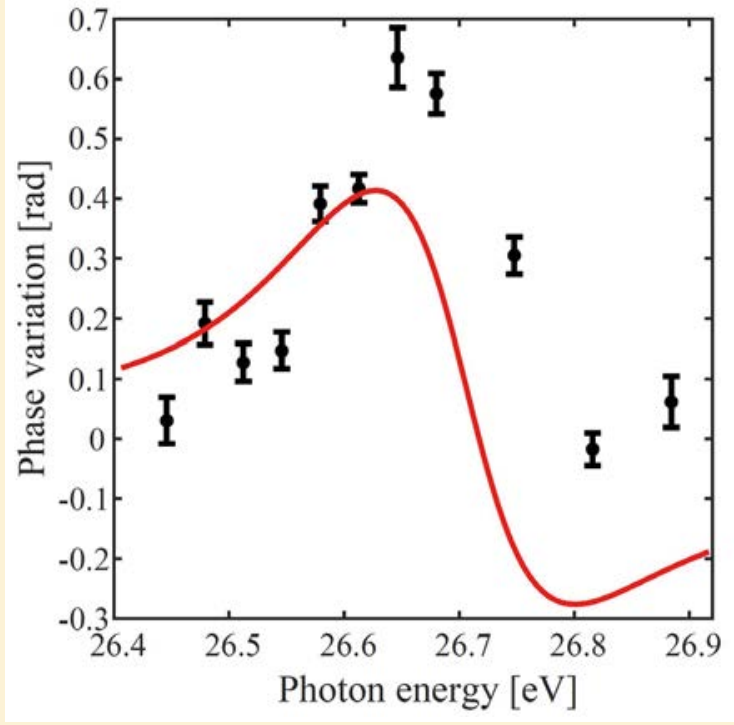

\section{L'OPTIQUE EST NOTRE AVENIR}

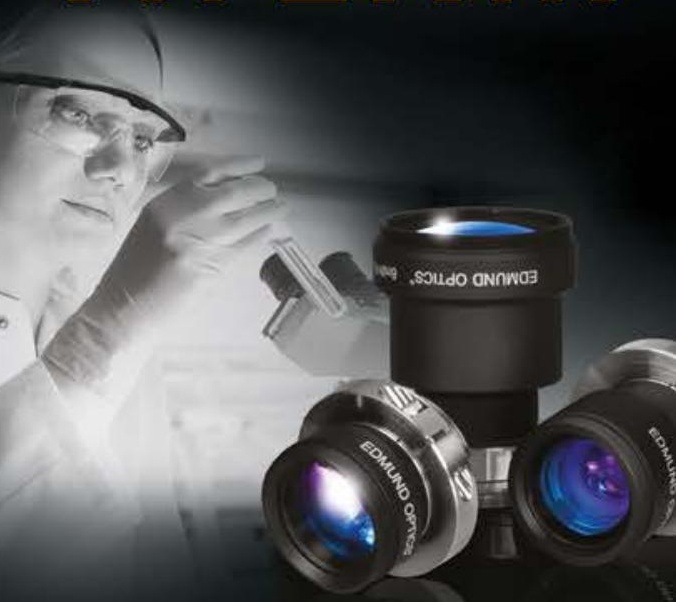

LOPTIQUE est une technologie habilitante, car elle rend possible plusieurs applications dans la fabrication avancée, la communication, la protection et la sécurité, les technologies numériques, l'énergie, la médecine, ainsi que les tests et mesures. Chez Edmund Optics ${ }^{\circ}$, nous visons à RENDRE LAVENIR POSSIBLE en nous concentrant sur l'avancement de tous les aspects de la vie et en surmontant les limites technologiques avec l'imagerie.

Pour en savoir plus

www.edmundoptics.fr/ future

Rendez-nous visite :
$24-27$ Avril 2018
Hall 4, Stand 4411
22 - 26 Avril 2018
Stand G524

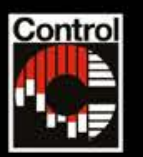

SPIE. PHOTONICS

sales@edmundoptics.fr

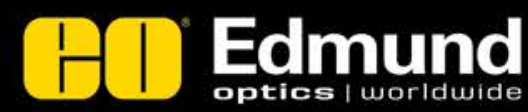




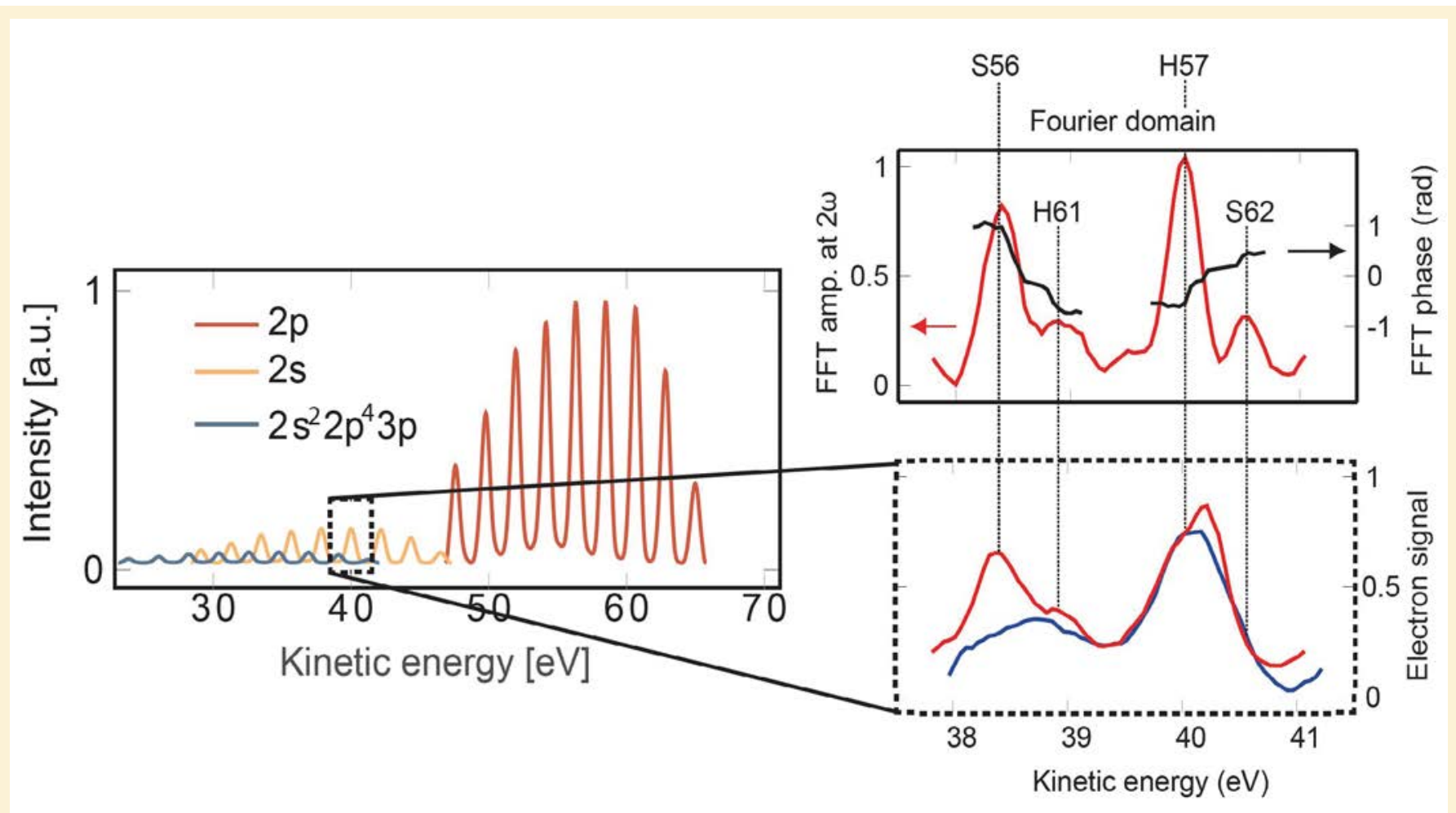

Figure 5. Illustration of the spectrally resolved RABBIT sideband analysis applied in [27]. The left graph shows simulated photoelectron spectra, obtained for attosecond pulse trains generated with $800 \mathrm{~nm}$ driving pulses, from the $2 p$ sub-shell in neon (red line, ionization potential $21.6 \mathrm{eV}$ ), from the $2 s$ sub-shell (yellow line, ionization potential $48 \mathrm{eV}$ ) and from ionization with shake-up (blue line, ionization potential $55.8 \mathrm{eV}$ ). The photoelectrons due to shake-up ionization lie between the ones from the $2 s$ sub-shell and would thus overlap with $2 s$ sidebands in a RABBIT measurement. The right side of the figure shows the photoelectron kinetic energy region around harmonic 57 and sideband 56 of the $2 s$ sub-shell. The lower plot shows the measured photoelectron spectrum for APTs only (blue line) and APTs+probe (red line), clearly visualizing the spectral overlap of sideband 56 with photoelectrons coming from ionization with shake up by absorption of harmonic 61 . The upper plot shows the oscillation amplitude (red line) and extracted phase (black line), obtained from Fourier analysis. Only the low energy part of sideband 56 (which is free from overlap) can be used to retrieve the sideband phase.

continuum, i.e. auto-ionize. In the experiment, a Fano resonance in argon is spectrally scanned with harmonic 17 of a $800 \mathrm{~nm}$ driving pulse. The phase of the resonance is imprinted on the adjacent sidebands and can be extracted using a sideband unaffected by the resonance as reference. The experimentally obtained phase is shown in Figure 4. This gives a characterization of the electron wave packet in amplitude and phase, which e.g. in the case of helium with a single continuum channel can be used to analyse it in the time-frequency domain $[25,26]$ similarly to what is done in ultrafast optics for ultra-short optical pulses, and provides intriguing complementary information to the spectral domain investigations extensively performed in the past.

\section{Conclusion}

To conclude our article, we would like to focus on a very recent result. Enabled by more reliable and long-term stable lasers as well as more efficient and high-resolution photoelectron spectrometers, the signal-to-noise ratio in RABBIT measurements can be increased to a level that spectral information can now often be obtained by directly analysing the sideband phase energy-resolved instead of averaging it over the whole width of the sideband [26]. This approach has helped to resolve the long-standing mystery of the $2 s / 2 p$ relative ionization delay in neon [27]. One condition for a RABBIT measurement to work properly is that the sidebands are not overlapping spectrally with any other states, which would lead to a wrong phase retrieval of the sideband. In the RABBIT traces recorded for photoelectrons from the $2 s$ and $2 p$ sub-shells in neon in [27], the $2 s$ sidebands were overlapping with peaks due to $2 p$ ionization with shake up of another $2 p$ state to the $3 p$ state, i.e. an ionization event where due to electron-electron interactions the ion is left in an exited state. However, the correct phase of the sideband could be obtained by only using the spectral interval where the sideband was free from overlap. Using this technique, relative ionization time delays between the $2 s$ and $2 p$ sub-shells in neon were obtained, now in excellent agreement with theory [27]. The energy-resolved sideband analysis is illustrated in Figure 5. 
The probably most exciting aspect of the techniques discussed in this article is the ability to measure amplitude and phase of electron wave packets and by that have full access to the temporal dynamics on the attosecond time scale. Modern laser technology in combination with high-resolution photoelectron spectrometers provide high temporal and energy resolution, opening the door to measurements on more complicated systems like molecules or nano-structures. Furthermore, laser technology currently pushes the development of HHG attosecond sources with higher photon energy in the range of hundreds of electron-volts. After almost two decades of studying mostly valence shell dynamics, attosecond science is now well prepared to also put its focus on the rich dynamics of the inner atomic shells. -

\section{FURTHER READING}

[1] A. McPherson et al.,J. Opt. Soc. Am. B 4, 595 (1987)

[2] M. Ferray et al., J. Phys. B 21, 31 (1988)

[3] P. Antoine et al.,Phys. Rev. Lett. 77, 1234 (1996)

[4] P.B. Corkum, Phys. Rev. Lett. 71, 1994 (1993)

[5] K.J. Schafer et al., Phys. Rev. Lett. 70, 1599 (1993)

[6] M. Lewenstein et al., Phys. Rev. A 49, 2117 (1994)

[7] H. Vincenti et al., Phys. Rev. Lett. 108, 113904 (2012)

[8] C.M. Heyl et al., New J. Phys. 16, 1367 (2014)

[9] F. Calegari et al., J. Phys. B: At. Mol. Opt. Phys. 45, 074002 (2012)

[10] C.M. Heyl et al., J. Phys. B: At. Mol. Opt. Phys. 50, 013001 (2017)

[11] P.M. Paul et al., Science 292, 1689 (2001)

[12] M. Hentschel et al., Nature 414, 509 (2001)

[13] R. López-Martens et al., Phys. Rev. Lett. 94 033001 (2005)
[14] E.P. Wigner, Phys. Rev. 98, 145 (1955)

[15] A.L. Cavalieri et al., Nature 449, 1029 (2007)

[16] M. Schultze et al., Science 328, 1658 (2010)

[17] K. Klünder et al., Phys. Rev. Lett. 106, 143002 (2011)

[18] D. Guénot et al., Phys. Rev. A 85 , 053424 (2012)

[19] M. Magrakvelidze et al., Phys. Rev. A 91, 063415 (2015)

[20] J.M. Dahlström et al., Chem. Phys. 414, $53(2013)$

[21] E.P. Månsson et al., Nature Phys. 10, 207 (2014)

[22] M. Swoboda et al., Phys. Rev. Lett. 104, 103003 (2010)

[23] M. Kotur et al.,Nature Comm. 7, 10566 (2016)

[24] U. Fano, Phys. Rev. 124, 1866 (1961)

[25] D. Busto et al.,J. Phys. B 51, 044002 (2018)

[26] V. Gruson et al., Science 354, 313 (2016)

[27] M. Isinger et al., Science 358, 893 (2017)

\section{Fibertech Lille (France)}

Fibertech Lille is a high technology research center based in the IRCICA institute in Lille (France). It is devoted to the design/fabrication of industry-standard passive and active optical fibers as well as state-of-the-art specialty optical fibers for research purposes. This platform gathers several original facilities dedicated to the fabrication of preforms by MCVD (Modified Chemical Vapor Deposition), OVD (Outside Vapor Deposition), Sol-GeL and powder processes as well as an area dedicated to develop original chemical precursors, allowing to address a large variety of dopants ( $\mathrm{Yb}, \mathrm{Er}, \mathrm{Bi}$, gold nanoparticles...) and glass geometries. Twosided fiber drawing tower is available $(12 \mathrm{~m}$ high) permitting the fabrication of any kind of state of the art silica optical fibers (spun fibers, photonic crystal fibers, hollow core fibers, large mode area fibers, rare-earth doped fibers, few mode fibers, multicore fibers...), with uniform or longitudinally-controlled profiles (tapers or topographic fibers). In addition, the platform is equipped with facilities for the characterization of fiber or preforms (preform index profiler, electronic microscope, OTDR, dispersion/nonlinearity measurement platforms, modal content with $\mathrm{S}^{2}$ method), the post processing and the functionalizing of optical fibers (e.g. writing of long fiber Bragg gratings or fabrication of specific beam combiners or mode adapters for high-power fiber laser systems). Fibertech Lille is composed of leading experts in the design/fabrication and implementation of complex optical fibers in lightwave systems for pure research

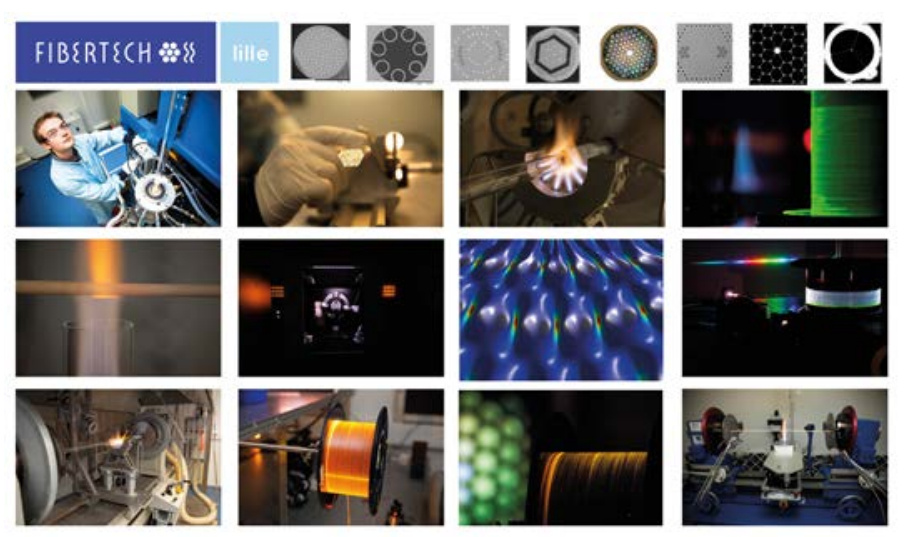

or applications -oriented purposes. The platform is driven by the photonics group (PHLAM laboratory, University of Lille) which main research interests are on fiber optics telecommunications, biophotonics, nonlinear optics (theory and experiments), fiber laser sources development in close collaboration with academic and industrial partners from all over the world. All these aspects make Fibertech Lille one of the leading expert in fiber optics in Europe.

\section{CONTACT \\ FIBERTECH LILLE \\ Géraud Bouwmans, Fibertech Lille leader, geraud.bouwmans@univ-lille1.fr \\ Arnaud Mussot, Photonics Group leader, arnaud.mussot@univ-lille1.fr \\ http://fibertech.univ-lille.fr/presentation}

